\title{
Roles and Responsibilities of Expert Clients in Adolescent HIV Service Delivery in Eswatini: A Conventional Content Analysis
}

Charisse Ahmed ( $\nabla$ cahmed@nursing.upenn.edu )

University of Pennsylvania https://orcid.org/0000-0003-1146-943X

\section{Guy Weissinger}

University of Pennsylvania

Anne Teitelman

University of Pennsylvania

Ndumiso Sabelo Dlamini

University Research Co LLC

Nontsikelelo Patience Dlamini

University Research Co LLC

Thuthukile Cebsile Dlamini

University Research Co LLC

Angel Nkambule

University Research Co LLC

Siphesihle Nkambule

University Research Co LLC

Bridgette M. Brawner

University of Pennsylvania

Marianne Calnan

University Research Co LLC

\section{Research}

Keywords: Lay health workers, expert clients, Eswatini, sub-Saharan Africa, adolescents, HIV/AIDS, qualitative research

Posted Date: December 9th, 2020

DOI: https://doi.org/10.21203/rs.3.rs-122032/v1

License: (c) (i) This work is licensed under a Creative Commons Attribution 4.0 International License.

Read Full License 


\section{Abstract}

\section{Background}

Like in other countries throughout sub-Saharan Africa, Eswatini employs a cadre of HIV-positive lay health workers known as expert clients (ECs) for the purpose of mitigating human resource gaps in HIV care. However, there are no insights in the literature describing the scope of their contribution to adolescent-specific HIV service delivery. Therefore, we aimed to elucidate the specific roles and responsibilities of ECs with regard to service delivery among adolescents living with HIV in Eswatini, and to provide recommendations for enhancing adolescent service provision among ECs and similar lay health workers throughout low- and middle-income countries.

Methods

An exploratory qualitative descriptive methodology using conventional content analysis was employed to meet our study aims. We recruited 20 expert clients and 12 key informants (i.e., program managers, program coordinators, and nurses) to participate in semi-structured interviews, and we arranged four focus group discussions among adolescents living with HIV with 7 to 10 participants per focus group.

Results

Adherence counseling in clinical and community settings was considered paramount to the roles and responsibilities of ECs with regard to adolescent-specific HIV service delivery. The following recommendations were made to enhance EC service delivery practices among adolescents: a) training in adolescent developmental, sexual, and reproductive needs, b) training to enhance clinical knowledge and skills, c) additional work equipment and compensation, and d) more parent and guardian engagement in their work.

Conclusions

While expert clients meet the needs of HIV-positive adolescents in several capacities, ECs require additional resources, skills, and training to improve their work, especially within the realm of sexual and reproductive health. Future research is needed to evaluate the impact of EC service delivery on adolescent health outcomes.

\section{Background}

Lay health workers (LHWs) are widely utilized to mitigate human resource gaps in HIV service delivery throughout low- and middle-income countries (LMICs), and especially within African nations $(1,2)$. Despite the proliferation of LHWs in sub-Saharan Africa, there remains a dearth of evidence regarding laydelivered services specifically for adolescents living with HIV (ALHIV) within this region (3-5). The scarcity of developmentally appropriate interventions for adolescents is particularly troubling due to the poor treatment outcomes faced by this population when compared to that of other age groups. ALHIV often 
undergo higher rates of medication non-adherence and subsequent virologic failure in comparison to children and adults (6-10). With the limited pool of formally trained clinicians to meet the unique needs of African youth, it is imperative to identify and expand sustainable service delivery interventions that can be safely and effectively delivered by LHWs for this population.

Eswatini (formerly known as Swaziland) is a low middle income country in sub-Saharan Africa with an adult HIV prevalence rate of $27 \%$ (11), and only 1.7 physicians and 16 nursing/midwifery personnel available per 10,000 population (12). In response to this human resource crisis, a cadre of LHWs known as expert clients (ECs) engage in task shifting to alleviate the workload of nurses in managing the significant burden of HIV disease in the country (13-15). ECs are HIV-positive adults and provide psychosocial support amongst seropositive clients who are 10 years of age and older (16-20). ECs often work alongside nurses to facilitate Teen Club, a peer support group widely available for ALHIV in countries throughout sub-Saharan Africa (21-23). Teen Club is an adolescent-friendly service delivery that is associated with improved retention in care and treatment adherence among ALHIV in resources-poor settings $(21,24)$. However, little is known about the specific roles and responsibilities of ECs in promoting the health and wellbeing of adolescents in and outside of the Teen Club milieu.

To expand the literature regarding lay-delivered HIV services for adolescents in resource-poor settings, our study aimed to (1) clarify the roles and responsibilities of ECs regarding adolescent-specific HIV service provision in Eswatini, and (2) provide recommendations for enhancing adolescent-specific service delivery practices among ECs. Given the scarcity of evidence-based lay-delivered interventions for ALHIV, an exploratory study will add to the body of knowledge regarding lay contributions to adolescent health and provide specific insights into enhancing and scaling up adolescent-specific HIV services in resourcelimited settings.

\section{Methods}

\section{Design and setting}

Qualitative descriptive studies are typically used to investigate health care and nursing-related phenomena and is most suitable when more information is desired to develop or refine an intervention (25). Hence, we decided to conduct an exploratory qualitative descriptive methodology using conventional content analysis to meet our study aims. We recruited three groups to participate in our study: 1) ECs, 2) ALHIV who were current participants of Teen Club, and 3) key informants (KIs) which included program managers, program coordinators, and nurses. Participants were recruited from the Hhhoho, Lubombo, and Manzini regions of Eswatini. ECs and ALHIV were conveniently sampled from Teen Club sites to participate in this study while snowball sampling was utilized to recruit KIs. Kls and ECs were recruited to participate in semi-structured interviews while ALHIV participated in focus group discussions. We conducted four focus group discussions at Teen Club sites and semi-structured interviews took place at a place most convenient for the participant, which was usually their workplace. Although 19 is typically the maximum age for Teen Club participation, adolescents may participate in the 
program until their early twenties. Therefore, our study included 20-year-old participants in the focus group discussions. In addition to the 32 adolescents who participated in the study, we interviewed 12 and $20 \mathrm{Kls}$ and ECs, respectively.

\section{Data collection}

Data collection occurred between April and May 2017. All focus groups were conducted in siSwati, the local language in Eswatini. EC and KI participants had the option of completing the interviews in English or siSwati. Interviews in English were conducted by the first author while siSwati interviews and focus group discussions were conducted by a Swazi research assistant (NSD) who also translated the interview guides from English to siSwati. All semi-structured interviews and focus group discussions were audio recorded and transcribed verbatim. Five research assistants (NSD, NPD, TCD, AN, and SN) transcribed the siSwati interviews and translated them to English. All siSwati transcripts were reviewed and translated in pairs by four research assistants (NPD, TCD, AN, and SN) and subsequently checked by alternate pairs to confirm accuracy. The first author transcribed the English interviews and one fourth of those transcripts were checked by the research team.

All semi-structured interviews consisted of open-ended questions to assess the roles and responsibilities of ECs regarding adolescent service delivery. Participants were also asked to provide recommendations for improving EC service delivery practices among adolescents. During the focus group discussions, adolescents were asked open-ended questions regarding their interactions and experiences with ECs as well as their perceptions of EC roles and responsibilities. KIs and ECs also received a questionnaire that comprised of close-ended questions regarding the health needs of ALHIV in sub-Saharan Africa that were met by ECs, and were based on nine categorical constructs introduced by Pettitt and colleagues (26). These constructs were: drug access and availability, clinical care, nutritional care, sexual and reproductive health, psychological support, social support, transition of care, resources, and advocacy. Pettit et al. conducted a multi-country assessment to identify the specific needs of ALHIV in sub-Saharan Africa and used these categorical constructs as a guiding framework for their study. We therefore utilized this framework to confirm the extent of which ECs meet the multifaceted needs of ALHIV in Eswatini.

\section{Data analysis and interpretation:}

Data analysis was carried out by the first and second authors, CVA and GW. NVivo 12 software was used to facilitate our conventional content analysis as recommended by Hsieh and Shannon (2005) (27). The qualitative data was inductively coded in vivo, and second level codes were subsequently produced to meaningfully categorize our data. To ensure inter-rater reliability, two authors (CVA and GW) were responsible for coding the data, and consensus of coding was established by a cumulative kappa score of at least 0.80 . To enhance the trustworthiness of our data, three study participants confirmed the relevance and accuracy of our findings from our content analysis in November to December 2020, a technique known as member checking (28). 


\section{Results}

Participant demographic characteristics are summarized in Table 1. The age of the focus group participants ranged from 10 to 20 years. Two focus groups comprised of older adolescents (i.e., aged 15 to 20 years) while the remaining two consisted of younger adolescents (i.e., aged 10 to 14 years) and older adolescents. Focus group participants included adolescents who acquired HIV vertically (40.6\%) and horizontally (53\%). The median age of the ECs and KIs were 35.5 and 36.5 , respectively.

\section{Table 1. Demographic characteristics of participants}

\begin{tabular}{|c|c|c|c|}
\hline & Adolescents living with HIV & Expert Clients ${ }^{a}$ & Key Informants ${ }^{b}$ \\
\hline Number of Participants & 32 (7 - 10 per focus group) & 20 & 12 \\
\hline Age range (median) & $10-20(15)$ & $22-43(35.5)$ & $25-48(36.5)$ \\
\hline Sex (\%) & Female (53.1) & Female (80) & Female (66.7) \\
\hline \multicolumn{4}{|c|}{$\begin{array}{l}\text { a. } 16 \text { ECs were facility-based (worked primarily in a health facility), } 3 \text { were community-based (workec } \\
\text { primarily in community settings), and } 1 \text { was community- and facility-based }\end{array}$} \\
\hline
\end{tabular}

\section{Roles and responsibilities of expert clients}

In the context of adolescent-specific service delivery, adherence support was considered paramount to the roles and responsibilities of ECs. Adherence support consisted of counseling, education, follow-up visits, and pill counts (i.e., monitoring adherence by calculating the number of pills taken since the last refill appointment). One EC referred to this type of work as client tracking, which was defined as "checking whether clients come to the health center at the right time and they have correct adherence." Communitybased ECs were primarily responsible for tracking clients who have missed a clinic appointment within seven to ninety days (i.e., defaulters) or over ninety days (i.e., lost to follow-up) by visiting their homes and, if reached, encouraging them to resume treatment and attend their appointments. On the other hand, facility-based ECs performed all of their work within a health facility, which included conducting counseling sessions and pill counts, following up with patients via phone call, and documenting patient information. Facility-based clients would refer defaulters to community-based ECs for home-based follow-up. Community-based ECs would also consult with other community health workers (e.g., rural health motivators) to locate defaulters or clients that were lost to follow-up. When participants were asked about the most important role of ECs in promoting the health of ALHIV, many agreed that EC support as role models and adherence supporters were pivotal in their work among this age group.

"I always ask for the names of defaulters from the [facility-based] expert client, talk to the adolescents' parents and find out why they are defaulting. I normally choose a time when I know that the adolescent will also be at home so that I can converse with both the parent and the adolescent if the adolescent is still young. I speak directly to the older adolescents and remind them that their date has passed but they 
failed to fetch their medication [and] then they remember and go to the health facility" (community-based EC, female, age 37)

Psychosocial support is another major component of the roles among ECs in serving ALHIV. As proposed by the participants, psychosocial support for seropositive adolescents may involve helping them navigate the unique psychosocial challenges they face such as orphanhood, suicide ideation, abuse, neglect, poverty, and stigma. Most participants agreed that ECs provided the same services to adolescents as they did for adults, but with a different approach. Participants stated that ECs have to approach adolescentspecific care with more patience, sensitivity, and attentiveness. Table 2 details the specific responsibilities of ECs which were derived from the interviews and are organized by Pettitt and colleagues' (2013) categorical constructs.

\section{Table 2. Description of services provided to ALHIV by ECs}

\begin{tabular}{|ll|}
\hline $\begin{array}{l}\text { Pettitt et al.'s } \\
(2013) \\
\text { Categorical } \\
\text { Constructs }\end{array}$ & Scope of Responsibilities among Expert Clients \\
\hline Clinical care & $\begin{array}{l}\text { Vital signs, pill counts, track defaulters, monitor viral load and } \text { CD }_{4} \text { count, } \\
\text { appointment reminders, weight checks, patient filing and documentation, } \\
\text { telephone follow-up, TB sputum collection* }\end{array}$ \\
\hline $\begin{array}{l}\text { Nutritional care } \\
\begin{array}{l}\text { HIV drug access } \\
\text { and availability }\end{array}\end{array}$ & Nutrition education*, food provision* \\
\hline $\begin{array}{l}\text { Sexual and } \\
\text { reproductive } \\
\text { health }\end{array}$ & Distribute condoms*, sex education* \\
\hline $\begin{array}{l}\text { Psychosocial } \\
\text { support }\end{array}$ & $\begin{array}{l}\text { Adherence counselling, HIV disclosure counselling, home visits, coping and life } \\
\text { skills }\end{array}$ \\
\hline $\begin{array}{l}\text { Resources } \\
\text { Advocacy }\end{array}$ & HIV education, referrals \\
\hline $\begin{array}{l}\text { Transition of } \\
\text { care }\end{array}$ & Liaisons between ALHIV and professional healthcare staff \\
\hline *ECs have varying degrees of skills, training, and/or resources to perform these tasks \\
\hline
\end{tabular}

\section{Recommendations}

\section{Training in adolescent developmental, sexual, and reproductive needs}

Growth and development training was frequently recommended by Kls and ECs for improving EC communication with adolescents at varying stages of development. Another common suggestion among 
KIs and ECs was the need for ECs to obtain more training, education, and resources pertaining to the sexual and reproductive health (SRH) needs of ALHIV. One KI participant suggested that ECs provide minimal education on family planning and SRH issues because "their main focus is on the adherence piece." One adolescent desired to be educated on "behaviors that [would] prevent the spread of HIV and protect others." As one KI suggested, sexual debut may occur as early as 12 years of age in Eswatini, thus requiring early conversations about SRH among ALHIV. However, several Kls and ECs suggested that some ECs are not equipped to handle SRH issues and are unable to provide condoms or information on family planning:

"We do not have a family planning section or condoms [in this facility] yet we do have adolescents who are already engaged in sexual intercourse; instead we refer the adolescents to places which we are not even sure if the adolescents really go" (facility-based EC, female, age 32).

"We need to train [ECs] on sexual [and] reproductive health because we also want to reduce teenage pregnancy amongst the adolescents living with HIV and we have seen that they do get pregnant and they do change partners as well. So we need to empower the expert clients also with SRH information" (program coordinator, female, age 29).

\section{Training to enhance clinical knowledge and skills}

$\mathrm{EC}$ and $\mathrm{KI}$ participants identified the importance of augmenting the clinical expertise of ECs. Although ECs are trained to collect sputum cultures for tuberculosis (TB) screenings, others desired more skills in the following areas to enhance their role: more frequent refresher trainings, more information regarding antiretroviral drug regimens, collection of vital signs, mid-upper arm mid-upper arm circumference (MUAC) measurements to assess nutrition status, education on cervical cancer risks, technological skills, a better understanding of plasma markers pertinent to HIV patients (i.e., HIV viral load and CD 4 T-cell measurements), and sign language to improve communication with ALHIV with hearing impairments. $\mathrm{KI}$ and EC participants recommended more EC collaboration with nurses and community health workers (i.e., rural health motivators). KI participants also recommended more supervision and evaluation of the work of ECs.

One nurse recommended time-efficient coordination efforts between clinicians and ECs with regard to TB testing by suggesting that ECs perform sputum collection while patients are queuing for their appointments to accelerate physician and nursing assessments. She also suggested for ECs to receive training on HIV testing so that they may test entire household members for HIV during routine home visits, and therefore make progress towards the country's goal of achieving the 90-90-90 targets[1]:

"As health workers, we promote that if you go to a homestead, even if I'm going to see a gogo [meaning grandmother] who has cancer, they have to assess the whole family. Are they tested for HIV? You remember 90-90-90, so we want all HIV-positive patients to know their status and we want them to be on ART and suppressed. So now if we send an expert client to a homestead, just to see, 'Ah, is my patient still in care, what happened, they didn't come to the clinic,' and then you see, you know, ten people [are] in the 
homestead and they don't even know their statuses. So, maybe if we can improve that, it would be better" (nurse, female, age 30).

\section{Additional work equipment and compensation}

Participants recommended equipment and other resources that would facilitate EC-delivered services for adolescents such as computers, private consultation rooms, and incentives. $\mathrm{KI}$ and EC participants recommended higher compensation for ECs that is commensurate with their workload. Participants from all groups recommended teaching aids (e.g., videos, posters, pamphlets) to guide their educational sessions. ECs were also in need of phone credit (i.e., airtime) in order to follow up with adolescents via phone call or text message on their personal cell phones. One EC suggested a transportation allowance for home visits since many of them do not have cars:

"Apparently [community-based ECs] walk to the homestead[s]... but that would limit the number of patients seen per day. So even the quality of the work [they do] would be compromised" (nurse, female, age 30).

Several participants wished that some of the adolescents' basic needs (e.g., food, clothing, toiletries) could be met through EC services. Although some ECs were able to provide adolescents with food, many of them lacked adequate resources to provide these necessities:

"Some of the adolescents come [to Teen Club] with say torn shoes or the only reason why they attend the Teen Clubs is [for] the free lunch which means that they do not benefit anything from the activities due to hunger and it hurts me when I cannot do anything to help them. I'm hoping that there will come a point when organizations will come and we will register the underprivileged and offer whichever assistance or even feed these adolescents" (facility-based EC, male, age 22).

"I would like to get some assistance from government and other organizations because some of these adolescents don't have basic needs like shelter, food, and clothing, and there is a lot of them that are still being discriminated and stigmatized. This is as a result of that they don't have parents at times and nobody cares when they are home, yes they do play with other children but it affects them when they play with kids who can wear nice clothes and they don't have, it affects them psychologically, in total I can say they are orphans" (nurse, male, age 34).

"I think so far the [ECs] are providing us with all the necessary help that we need but... we are from different homesteads with different challenges; for instance, as girls we need some sanitary pads and you find that we can't afford them so I was thinking that maybe if the [ECs] could provide us with pads maybe every month that could help a lot" (focus group participant).

It was suggested that psychosocial support, while a key role among ECs, is another aspect of service delivery that may be improved. Some recommended that ECs should acquire more knowledge and skills around behavior management and counseling. Adolescents suggested that they would benefit from more adherence support provided by ECs via counseling and reminders. A few participants across all groups 
suggested more counseling sessions, follow-ups, and home visits as a mechanism for enhancing psychosocial support provided to ALHIV by ECs:

"I think [the ECs] should have ample time and sit the adolescent[s] down and find out as to how is their lives at home... because I believe there are so many adolescents who can actually voice out that their home situation is not good" (focus group participant).

Additionally, there are other supplies that ECs may need to improve the involvement of adolescents in Teen Clubs such as equipment for recreational activities:

"Our main challenge is that we do not have enough equipment [at Teen Club] so we sometimes have to come up with games from nowhere" (focus group participant).

"The games [the adolescents] play are not enough, I think they need games like soccer which means they need soccer balls [and] skipping ropes, it shouldn't be that they play chasing games all the time, it has to change" (facility-based EC, female, age 35).

\section{More parent and guardian engagement}

Although ECs have been successful at involving some parents and guardians in their work, it was often unclear from the interviews the extent of rapport built between them. Several participants suggested for ECs to develop deeper relationships with the parents/guardians of ALHIV:

"Because an expert client is someone that the caregiver is in contact with most of the time, I think it would be critical for the expert client to also play [a] special role with the caregiver as well as the adolescent" (program coordinator, female, age 33).

\section{Discussion}

Our research demonstrates that ECs are significant contributors to the health and wellbeing of ALHIV in Eswatini. In addition, our findings provide specific insights into the evolving use of LHWs with regard to adolescent health, and therefore filling knowledge gaps regarding lay-delivered services available for ALHIV in sub-Saharan Africa. Moreover, our study offers practical recommendations for improving adolescent-specific HIV service delivery that may be applied to nonspecialized health workers throughout LMICs. Particularly in Eswatini, adolescents are less likely to achieve HIV viral suppression when compared to their adult counterparts, irrespective of adherence counseling provided by lay counselors (30). However, our study suggests that ECs, by virtue of their job description, are suitable cadres of LHWs for improving adherence and retention in care among ALHIV. For instance, a cadre of peer health workers known as community adolescent treatment supporters, with similar responsibilities as ECs, were found to effectively improve treatment adherence, retention in care, and psychosocial wellbeing among ALHIV in rural Zimbabwe (31). Therefore, future research should investigate the extent to which ECs and similar cadres of LHWs improve treatment outcomes among ALHIV in resource-poor settings. 
There has been much debate regarding the placement of LHWs in the larger scope of service delivery in LMICs, given that many LHWs are not well integrated into national health systems (32-34). De Neve and colleagues conducted a qualitative study in four Southern African countries, including Eswatini, which confirms the challenges of harmonizing LHW programs with national service delivery models (35). In corroboration of De Neve's study as well as other studies done in LMICs $(1,34)$, our research suggests that ECs need additional support, recognition, incentives, and training to strengthen and sustain their work. Furthermore, ECs are in need of additional resources to carry out their job responsibilities (e.g., phone credit, recreational supplies). Hence, additional governmental and nongovernmental funding and support is needed to leverage their work. As highlighted in a qualitative study evaluating the performance of LHWs in Eswatini (16), higher monetary compensation may also provide ECs with an increased impetus to perform their tasks efficiently.

This study does not provide answers to the nuanced experiences that adolescents face in their communication with ECs. Such insight may further assist researchers and policymakers with a more appropriate and reasonable plan for intervention development shaped to enhance adolescent service delivery. As task shifting begins to expand the skillset and scope of practice among ECs, it is important to consider strategies to assuage their work burden. Dlamini-Simelane and Moyer's (2017) ethnographic study confirms that while ECs have been able to take on more clinical responsibilities, the quality of care has not improved (36). Therefore, there is a need to consider robust strategic planning around coordinating responsibilities among professionally trained clinicians and lay personnel to avoid burnout and fragmented service provision that may inadvertently lead to inefficient time and costs spent on HIV service delivery. As suggested by Hermann and colleagues (37), without adequate supervision and continual training of LHWs, the quality of their work may diminish

Although adolescent-friendly HIV services are generally inadequate throughout resource-limited settings $(3,26,38)$, the model of care ascribed to ECs may be a plausible solution to this service delivery deficit.

However, addressing the SRH needs of ALHIV still remains a challenge in LMICs as well as in high-income countries (39-41). Adolescents in Eswatini are susceptible to gender-based violence, high fertility, and early sexual debut, and yet there are limited SRH and family planning services specifically for Swazi youth (42). Studies conducted throughout sub-Saharan Africa confirm that ALHIV have suboptimal treatment adherence and less than $30 \%$ of Swazi adolescents are using condoms amongst their sexual partners, thereby increasing transmission risks among this age group $(43,44)$. However, ECs may be able to learn from effective interventions implemented in sub-Saharan Africa to reduce sexual risk behaviors among adolescents $(45,46)$.

\section{Limitations}

A possible study limitation is respondent bias such as acquiescence and social desirability by our participants. Although measures were in place to keep the survey administration and focus group discussions as uniform as possible, there may be some variation in the way in which the research team administered, transcribed, and/or recorded the data. Additionally, there was no back translation of the 
transcripts and survey instruments, thus limiting the internal validity of our findings. Due to our convenience sampling method, the results may not be transferrable to other contexts or settings. However, it is possible that these findings may be transferable within Eswatini due to its small size and the homogeneity of service delivery practices throughout the country. Furthermore, ALHIV who do not participate in Teen Clubs were excluded from study participation, which further limits the transferability of our study.

\section{Conclusions}

Our study is the first to explore the roles and responsibilities of ECs in the provision of adolescent-specific HIV service delivery in Eswatini. While ECs demonstrate the capacity to respond to a wide array of needs for ALHIV, there still remain gaps in their services, especially within the arena of sexual and reproductive health. Further research is needed to explore the effect of EC service delivery on health outcomes among Swazi adolescents.

\section{Declarations}

\section{Ethics approval and consent to participate}

This research study was approved by Eswatini's National Health Research Review Board as well as the University of Pennsylvania's Institutional Review Board. Each participant was offered a consent form (if over the age of 18) or an assent form (if under the age of 18). To ensure informed consent/assent, a member of the research team described the study to each participant prior to obtaining the participant's written consent/assent. Adolescents under the age of 18 were able to participate if at least one parent or guardian was able to provide the research team with written informed consent. Parents and guardians were contacted by expert clients and were required to provide in-person informed consent prior to their child's study participation in the focus group discussions.

\section{Consent for publication}

All participants agreed to and signed written informed consent forms confirming their consent to publish our findings.

\section{Availability of data and materials}

Although our data is not available for the public, individuals interested in reviewing our data may contact the corresponding author.

\section{Competing interests}

The authors declare that there are no competing interests associated with our work.

\section{Funding}


This research study was funded by the Fulbright U.S. Student Program, a U.S. Department of State program that is sponsored by the U.S. Government and administered by the Institute of International Education, Inc. This study was implemented under the support of University Research Co., LLC in Mbabane, Eswatini who provided transportation for data collection, printers and printing materials, computers, and office space. Data analysis software was provided by the University of Pennsylvania School of Nursing.

\section{Authors' contributions}

The first author (CVA) planned and implemented this study under the guidance of the senior author (MC). All authors contributed to the data analysis plan for this study while CVA and GW carried out the analysis under the guidance of AT and BB. NSD, NPD, TCD, AN, and SN contributed to data collection, transcription of interviews, and translation of siSwati transcripts to English. CVA also contributed to data collection.

\section{Acknowledgements}

We would like to thank all of our study participants, and especially the people living with HIV who have generously participated and supported this research. We acknowledge the Ministry of Health's Swaziland National AIDS Programme (SNAP), especially Dr. Nomthandazo Lukhele and Ms. Nompilo Gwebu, who supported the involvement of expert clients in this research. Lastly, we thank the University Research Co., LLC, especially Dr. Samson Haumba, for partnering with us by providing several supplies and resources needed to conduct this research.

\section{References}

1. Mwai GW, Mburu G, Torpey K, Frost P, Ford N, Seeley J. Role and outcomes of community health workers in HIV care in sub-Saharan Africa: a systematic review. J Int AIDS Soc. 2013;16(1):18586.

2. Schneider $\mathrm{H}$, Okello $\mathrm{D}$, Lehmann $\mathrm{U}$. The global pendulum swing towards community health workers in low- and middle-income countries: a scoping review of trends, geographical distribution and programmatic orientations, 2005 to 2014. Human Resources for Health. 2016;14(1):65.

3. Koon AD, Goudge J, Norris SA. A review of generalist and specialist community health workers for delivering adolescent health services in sub-Saharan Africa. Human Resources for Health. 2013;11(1):54.

4. Murray KR, Dulli LS, Ridgeway K, Dal Santo L, Darrow de Mora D, Olsen P, et al. Improving retention in HIV care among adolescents and adults in low- and middle-income countries: A systematic review of the literature. PLoS One. 2017;12(9):e0184879.

5. Ridgeway K, Dulli LS, Murray KR, Silverstein H, Dal Santo L, Olsen P, et al. Interventions to improve antiretroviral therapy adherence among adolescents in low- and middle-income countries: A systematic review of the literature. PLoS One. 2018;13(1):e0189770.

6. Boerma RS, Bunupuradah T, Dow D, Fokam J, Kariminia A, Lehman D, et al. Multicentre analysis of second-line antiretroviral treatment in HIV-infected children: adolescents at high risk of failure. J Int 
AIDS Soc. 2017;20(1):21930.

7. Kranzer K, Bradley J, Musaazi J, Nyathi M, Gunguwo H, Ndebele W, et al. Loss to follow-up among children and adolescents growing up with HIV infection: age really matters. J Int AIDS Soc. 2017;20(1):21737.

8. Lamb MR, Fayorsey R, Nuwagaba-Biribonwoha H, Viola V, Mutabazi V, Alwar T, et al. High attrition before and after ART initiation among youth (15-24 years of age) enrolled in HIV care. Aids. 2014;28(4):559-68.

9. Nachega JB, Hislop M, Nguyen H, Dowdy DW, Chaisson RE, Regensberg L, et al. Antiretroviral therapy adherence, virologic and immunologic outcomes in adolescents compared with adults in southern Africa. J Acquir Immune Defic Syndr. 2009;51(1):65-71.

10. Adejumo OA, Malee KM, Ryscavage P, Hunter SJ, Taiwo BO. Contemporary issues on the epidemiology and antiretroviral adherence of HIV-infected adolescents in sub-Saharan Africa: a narrative review. Journal of the International AIDS Society. 2015;18(1):20049-.

11. Bicego GT, Nkambule R, Peterson I, Reed J, Donnell D, Ginindza H, et al. Recent patterns in population-based HIV prevalence in Swaziland. PLoS One. 2013;8(10):e77101.

12. World Health Organization. World health statistics 2015. Geneva: World Health Organization; 2015.

13. Sibbald B. HIV prevention: new pilots for beleaguered Swaziland. The Lancet. 2013;381(9861):103-4.

14. Sibbald B. Responding to Swaziland's dual epidemic. CMAJ. 2013;185(1):E13-4.

15. East Central and Southern African Health Community. Task shifting in Swaziland: A case study. Washington, DC: Futures Group, Health Policy Initiative, Task Order 1: East, Central, and Southern African Health Community (ECSA-HC); 2010. Contract No.: May 9.

16. Geldsetzer P, De Neve JW, Boudreaux C, Bärnighausen T, Bossert TJ. Improving the performance of community health workers in Swaziland: findings from a qualitative study. Hum Resour Health. 2017;15(1):68.

17. Tenthani L, Cataldo F, Chan AK, Bedell R, Martiniuk ALC, van Lettow M. Involving expert patients in antiretroviral treatment provision in a tertiary referral hospital HIV clinic in Malawi. BMC Health Services Research. 2012;12(1):140.

18. Tafuma TA, Mahachi N, Dziwa C, Marowa P, Moga T, Chimbidzikai T, et al. Time taken to link newly identified HIV positive clients to care following a home-base index case HIV testing: Experience from two provinces in Zimbabwe. PLoS One. 2018;13(8):e0201018.

19. Kyakuwa M, Hardon A, Goldstein Z. "The Adopted Children of ART": expert clients and role tensions in ART provision in Uganda. Med Anthropol. 2012;31(2):149-61.

20. Kyakuwa M. More hands in complex ART delivery? Experiences from the expert clients initiative in rural Uganda. African Sociological Review/Revue Africaine de Sociologie. 2009;13(1).

21. MacKenzie RK, van Lettow M, Gondwe C, Nyirongo J, Singano V, Banda V, et al. Greater retention in care among adolescents on antiretroviral treatment accessing "Teen Club" an adolescent-centred 
differentiated care model compared with standard of care: a nested case-control study at a tertiary referral hospital in Malawi. Journal of the International AIDS Society. 2017;20(3):e25028.

22. Midtb $\varnothing$ V, Shirima V, Skovdal M, Daniel M. How disclosure and antiretroviral therapy help HIV-infected adolescents in sub-Saharan Africa cope with stigma. Afr J AIDS Res. 2012;11(3):261-71.

23. Agarwal M, Van Lettow M, Berman J, Gondwe C, Mwinjiwa E, editors. Evaluation of a specialized psychosocial support intervention 'Teen Club'in improving retenion among adolescents on antiretroviral treatment (ART) at a tertiary referral hospital in Malawi. Oral presentation. 7th IAS conference on HIV pathogenesis treatment and prevention Kuala Lumpur; 2013.

24. McBride K, Parent J, Mmanga K, Chivwala M, Nyirenda MH, Schooley A, et al. ART Adherence Among Malawian Youth Enrolled in Teen Clubs: A Retrospective Chart Review. AIDS Behav. 2019;23(9):262933.

25. Kim H, Sefcik JS, Bradway C. Characteristics of Qualitative Descriptive Studies: A Systematic Review. Res Nurs Health. 2017;40(1):23-42.

26. Pettitt ED, Greifinger RC, Phelps BR, Bowsky SJ. Improving health services for adolescents living with HIV in sub-Saharan Africa: a multi-country assessment. Afr J Reprod Health. 2013;17(4 Spec No):1731.

27. Hsieh HF, Shannon SE. Three approaches to qualitative content analysis. Qual Health Res. 2005;15(9):1277-88.

28. Birt L, Scott S, Cavers D, Campbell C, Walter F. Member Checking: A Tool to Enhance Trustworthiness or Merely a Nod to Validation? Qual Health Res. 2016;26(13):1802-11.

29. Levi J, Raymond A, Pozniak A, Vernazza P, Kohler P, Hill A. Can the UNAIDS 90-90-90 target be achieved? A systematic analysis of national HIV treatment cascades. BMJ global health. 2016;1(2):e000010.

30. Jobanputra K, Parker LA, Azih C, Okello V, Maphalala G, Kershberger B, et al. Factors associated with virological failure and suppression after enhanced adherence counselling, in children, adolescents and adults on antiretroviral therapy for HIV in Swaziland. PLoS One. 2015;10(2):e0116144.

31. Willis N, Milanzi A, Mawodzeke M, Dziwa C, Armstrong A, Yekeye I, et al. Effectiveness of community adolescent treatment supporters (CATS) interventions in improving linkage and retention in care, adherence to ART and psychosocial well-being: a randomised trial among adolescents living with HIV in rural Zimbabwe. BMC Public Health. 2019;19(1):117-.

32. Mundeva H, Snyder J, Ngilangwa DP, Kaida A. Ethics of task shifting in the health workforce: exploring the role of community health workers in HIV service delivery in low- and middle-income countries. BMC Med Ethics. 2018;19(1):71.

33. Schneider H, Lehmann U. Lay health workers and HIV programmes: implications for health systems. AIDS Care. 2010;22 Suppl 1:60-7.

34. Pallas SW, Minhas D, Pérez-Escamilla R, Taylor L, Curry L, Bradley EH. Community health workers in low- and middle-income countries: what do we know about scaling up and sustainability? Am J Public Health. 2013;103(7):e74-82. 
35. De Neve J-W, Garrison-Desany H, Andrews KG, Sharara N, Boudreaux C, Gill R, et al. Harmonization of community health worker programs for HIV: a four-country qualitative study in Southern Africa. PLoS medicine. 2017;14(8).

36. Dlamini-Simelane T, Moyer E. Task shifting or shifting care practices? The impact of task shifting on patients' experiences and health care arrangements in Swaziland. BMC Health Services Research. 2017;17(1):20.

37. Hermann K, Van Damme W, Pariyo GW, Schouten E, Assefa Y, Cirera A, et al. Community health workers for ART in sub-Saharan Africa: learning from experience-capitalizing on new opportunities. Human resources for health. 2009;7(1):31.

38. James S, Pisa PT, Imrie J, Beery MP, Martin C, Skosana C, et al. Assessment of adolescent and youth friendly services in primary healthcare facilities in two provinces in South Africa. BMC health services research. 2018;18(1):809-.

39. Okawa S, Mwanza-Kabaghe S, Mwiya M, Kikuchi K, Jimba M, Kankasa C, et al. Sexual and reproductive health behavior and unmet needs among a sample of adolescents living with HIV in Zambia: a cross-sectional study. Reprod Health. 2018;15(1):55.

40. Hamzah L, Hamlyn E. Sexual and reproductive health in HIV-positive adolescents. Curr Opin HIV AIDS. 2018;13(3):230-5.

41. Munea AM, Alene GD, Debelew GT. Quality of youth friendly sexual and reproductive health Services in West Gojjam Zone, north West Ethiopia: with special reference to the application of the Donabedian model. BMC Health Serv Res. 2020;20(1):245.

42. Dlamini BR, Mabuza P, Masangane Z, Silindza T, Dlamini M, Dlamini P. The HIV and Sexual Reproductive Health Status of Young People in Swaziland: the rationale for focused youth investment. Journal of AIDS and Clinical Research. 2017;8(3):669.

43. Zgambo M, Kalembo FW, Mbakaya BC. Risky behaviours and their correlates among adolescents living with HIV in sub-Saharan Africa: a systematic review. Reproductive Health. 2018;15(1):180.

44. Government of the Kingdom of Eswatini. Swaziland HIV Incidence Measurement Survey 2 (SHIMS2) 2016-2017. Mbabane: Government of the Kingdom of Eswatini; 2019.

45. Jemmott LS, Jemmott JB, 3rd, Ngwane Z, Icard L, O'Leary A, Gueits L, et al. 'Let Us Protect Our Future' a culturally congruent evidenced-based HIV/STD risk-reduction intervention for young South African adolescents. Health Educ Res. 2014;29(1):166-81.

46. Pretorius L, Gibbs A, Crankshaw T, Willan S. Interventions targeting sexual and reproductive health and rights outcomes of young people living with HIV: a comprehensive review of current interventions from sub-Saharan Africa. Glob Health Action. 2015;8:28454. 